\title{
Como as tecnologias são incorporadas na educação e os seus potenciais ganhos na relação ensino/aprendizagem por meio da teoria da carga cognitiva
}

\author{
How technology is integrated into education and its potencial gains in a teaching/learning \\ relationship through cognitive load theory
}

SCAMATI, Vagner ${ }^{1}$

\begin{abstract}
Different types of technology are integrated into education in order to improve the teaching/learning relationship and also to provide a potential gain in the student learning process. In immersive virtual teaching environments it is possible to provide greater student engagement and interaction. This paper presents a systematic literature review on how the main technologies are applied at different educational levels and areas, as well as the effects of the mental load and the mental effort for the storage of instructions through the cognitive load theory.

Keywords: Education and technology, immersive learning environments, cognitive load

Resumo

Tecnologias mistas são incorporadas a educação com o objetivo de melhorar a relação ensino/aprendizagem e proporcionar um potencial ganho no proceso de aprendizagem do aluno. Em ambientes de ensinos virtuais imersivos é possivel proporcionar um maior engajamento e interação do aluno. Este trabalho mostra uma revisão sistemática da literatura de como as principais tecnologías são aplicadas nos diversos niveis educacionais e áreas, bem como o impacto do esforço da carga mental para o armazenamento das instruções por meio da teoría da carga cognitiva.

Palavras chave: Educação e tecnologia, ambientes imersivos aprendizagem, carga cognitiva
\end{abstract}

\section{Introdução}

Desde que a pandemia do Covid 19 se instalou no mundo e foi declarado pela Organização Mundial da Saúde (OMS), emergência em saúde pública, milhões de alunos se encontram fora da escola. As ações necessárias ao combate do novo corona vírus interromperam as aulas presenciais, criando graves consequências para a educação, afetando o calendário e a qualidade no ensino.

Uma das possibilidades para ajudar neste período, principalmente retomar a aprendizagem, contato e interação entre professore e alunos, foi promover o uso de mídias e seus recursos tecnológicos à educação. Atravessa-se um momento conturbado de debates, diagnósticos e indagações sobre o uso de tecnologias na educação. Dúvidas de como incorporar as tecnologias e como obter ganho nesta relação de ensino/aprendizagem

\footnotetext{
${ }^{1}$ Professor. IFPR - Instituto Federal do Paraná - Campus Palmas (PR). Curso Sistemas de Informação Brasil. Email: vagner.scamati@ifpr.edu.br
} 
inovadora, são recorrentes perante os atores da educação. É um momento sensível e que nos conduz a ressignificar a educação na perspectiva ensino/aprendizagem para propor, avaliar e discutir novos caminhos para a aprendizagem e inevitavelmente refletir sobre formas de como alunos terão que "aprender a aprender" na perspectiva de auto reorganizar a educação. Nesse cenário uma das opções que ganha espaço é a heutagogia.

Segundo (Hase \& Kenyon, 2001) a heutagogia não trata diretamente da relação ensino-aprendizagem, mas o foco se desloca para aprendizagem, é auto determinada pelo aluno, ele é quem define como e quando aprender. O professor fornece recursos por meio de tecnologias para a aprendizagem. A proposta é que o aluno seja o ator principal no processo de aprendizagem e no seu ritmo faça suas atividades educacionais.

Para que a educação se torne tecnológica em seus ambientes, diversos tipos de tecnologias diferentes podem ser utilizados. O conceito de realidade mista ou misturada (RM) abrange diversos outros tipos de realidade. Segundo (Speicher, Hal, \& Nebeling, 2019) , a maioria de autores propõe a noção de realidade mista abrangendo genericamente tudo que se encontra no chamado contínuo da realidade criado por (Miligram \& Kishino, 1994), ou seja, a RM abarca a realidade virtual (RV) e a realidade aumentada (RA), combinando o mundo real e o virtual - virtualidade aumentada.

Na RM é possível criar Ambientes Virtuais de Aprendizagem (AVA), que são ambientes integrados de informação, comunicação e aprendizagem online, que buscam aperfeiçoar a qualidade de ensino dos alunos, por meio da elaboração de atividades fora do ambiente das aulas presenciais. Segundo (Tori \& Hounsell, 2018), eles permitem integrar diferentes mídias e recursos, apresentar informações de maneira organizada, desenvolver interações entre pessoas e objetos de aprendizagem, elaborar e socializar produções tendo em vista atingir determinados objetivos e proporcionar a interação entre seus usuários. Eles podem ser imersivos ou não imersivos.

A imersão do ambiente está relacionada a sensação na utilização da RV por meio de seus dispositivos. Ambientes totalmente imersivos refere-se ao uso de um dispositivo conhecido como headset, que pode ser colocado na cabeça do aluno ou professor, um dos dispositivos pode ser o OHMD (optical head mounted display). Ele é usado para explorar o cenário e espaço tridimensional que permite ao aluno mover ou usar fisicamente um controle com sensores para rastreamento e utilizar nas mãos para o movimento e interagir em um ambiente virtual 3D (tridimensional) (Lee \& Wong, 2014). Ambientes não imersivo, também conhecido como desktop, é representado por um dispositivo que não seja um headset OHMD. Envolve o acesso a um ambiente virtual por de imagens e/ou vídeos em 360 graus ou outros ambientes 3D usando um computador de mesa e um monitor com periféricos de controle como joystick (controle com alavancas usados em jogos), mouse ou luvas especiais para controlar e explorar o movimento.

Usar as tecnologias trazem imensos desafios a educação, empregá-las ao processo de ensino/aprendizagem a diferentes níveis e áreas da educação, desde a escola básica até a universidade vem sendo discutido há tempo, sobretudo para oferecer possíveis resultados no ganho da aprendizagem. Um dos desafios é proporcionar ao aluno o engajamento, a interação e o uso colaborativo de forma satisfatória. Aplicações mais simples ou complexas, ambientes bidimensional ou tridimensional com aprendizagem imersiva ou semi imersiva, mundos virtuais, hologramas, aplicações web, e outras formas de tecnologias são empregados a favor da educação. 0 modo como a tecnologia educacional é usada e como é incorporada à experiência de aprendizagem é fundamental para sua eficácia.

Este trabalho apresenta uma revisão sistemática da literatura (RSL), acerca de ambientes de aprendizagem imersivos, semi imersivos e/ou aumentados, colaborativos (ou não), mas que utilizam a tecnologia de RM com propósitos distintos aplicados e incorporados em qualquer nível de educação e que podem (ou não) colaborar com o ganho de aprendizagem por meio da teoria da carga cognitiva. 


\section{Metodologia}

Para a elaboração dos critérios de busca nas bases de dados bibliográficos, segundo (Wazlawick, 2014) é necessário a montagem de uma estratégia de busca que envolve um conjunto de procedimentos com o objetivo de localizar a informação. Para a elaboração dos critérios de busca investigativa para esse trabalho foram definidas as Questões de Pesquisa (QP), as quais são apresentadas na Tabela 1.

Tabela 1

Questões de Pesquisa

\begin{tabular}{|c|l|}
\hline $\begin{array}{c}\text { Questões de } \\
\text { Pesquisa (QP) }\end{array}$ & \multicolumn{1}{c|}{ Descrição } \\
\hline QP1 & $\begin{array}{l}\text { Como são incorporados os ambientes de aprendizagem imersivos a qualquer nível educacional e } \\
\text { como são aplicados os tipos de realidades diferentes (realidade aumentada, realidade virtual, } \\
\text { realidade mista)? }\end{array}$ \\
\hline QP2 & Como as teorias de aprendizagem são utilizadas e relacionados com os ambientes de aprendizagem? \\
\hline QP3 & $\begin{array}{l}\text { Como é a relação entre o ambiente aprendizagem tecnológico e o ensino por meio da teoria da carga } \\
\text { cognitiva? }\end{array}$ \\
\hline
\end{tabular}

Fonte: Autor

\subsection{Estratégia de Busca}

Para a elaboração dos critérios de busca construiu-se strings de busca que foram executadas em tipos diferentes de base de dados, como trabalhos em conferências, jornais ou revistas específicas. Foram utilizadas 3 bases de dados por possuírem vasto volume de estudos publicados em periódicos e conferências da área de Tecnologia da Informação e Comunicação, e Educação: Digital library ACM - Digital Library IEEE Xplore - ERIC (Institute of Education e Sciences). A tabela 2 mostra os anais das Conferências e Periódicos utilizados na pesquisa manual.

\subsection{String de Busca}

O conjunto de critérios de acordo com (Madhukar, et al., 2004), foi utilizada para a definição das strings de busca, que consiste em: P (população), estabelece o foco da pesquisa; I (intervenção), delimita o foco da pesquisa dentro de um escopo mais amplo; $C$ (comparação), identifica alternativas e compara com a delimitação realizada na intervenção; e $\mathrm{O}$ (resultados /saídas), lista o que se pretende realizar, medir, melhorar ou afetar em relação à população.

Assim, os critérios definidos foram: a) População: a população definida foram os ambientes virtuais de aprendizagem. Para procurar a população, foram utilizadas as palavras-chave immersive education environment; b) Intervenção: a intervenção visou identificar e apresentar os mais relevantes métodos, tecnologias e mecanismos de promoção de ambientes de aprendizagem imersivos de RM. Para isso foram utilizadas as palavras-chave: mixed reality; c) Comparação: o foco do estudo não abrangeu a realização de estudos comparativos, sendo assim, tal técnica não foi utilizada na estratégia de pesquisa e na formação das strings de busca; d) Resultado: procurou-se por trabalhos relacionados tanto à ambientes de aprendizagem embasados em teoria de aprendizagem e relacionados com a teoria da carga cognitiva de forma aberta para qualquer nível de educação. Assim, foram avaliados trabalhos que continham palavras-chave: leanirg theory OR cognitive load theory.

A partir da definição desses critérios definiu-se a seguinte string de busca que foi aplicada, sempre que possível, para apresentar os resultados da busca em cima tanto de título e palavras-chave: (("immersive education environment") and ("mixed reality") and ("learning theory" OR "cognitive load theory")) 


\subsection{Critérios de Seleção (Inclusão e Exclusão)}

Os seguintes critérios de seleção foram definidos para a seleção dos estudos primários: a)Trabalho deve estar disponível nas bases de dados digitais previamente definida e o ano de publicação dos estudos deve estar entre 2015 e 2020; b)Estudo deve ter sido escrito em inglês ou português, e deve propor ou avaliar metodologias, tecnologias de RM ou mecanismos de ambiente de aprendizagem imersivos ou semi imersivos incorporados na educação para qualquer tipo e área de nível educacional.

Como critério de exclusão dos estudos considerou-se o não atendimento de algum dos critérios de inclusão, como também: Tratar-se de trabalhos incompletos - publicados como Short Paper. Não apresentar informações suficientes para a extração dos dados esperados (Petersen, Vakkalanka, \& Kuzniarz, 2015).

\subsection{Estratégia de seleção}

A avaliação da qualidade dos estudos identificados após a execução da estratégia de busca, possibilitou a seleção dos artigos mais relevantes, sendo executada através dos passos de seleção mostrados anteriormente, os quais são apresentados na Figura 1. Os passos adotados na estratégia de busca foram: (1) Execução da estratégia de pesquisa envolvendo as buscas automática e manual. Com isso uma lista preliminar de estudos foi gerada e descartados os trabalhos duplicados; (2) Identificação de estudos potencialmente relevantes, com base na leitura do título e resumo. Nesse passo foi possível descartar estudos que são claramente irrelevantes para a pesquisa. Em caso de dúvida sobre a permanência de algum estudo, o próximo passo auxilia nesta definição; (3) Leitura da introdução, metodologia e conclusão dos trabalhos pré-selecionados, aplicando novamente os critérios de inclusão e exclusão; (4) Os trabalhos que forem selecionados na etapa 3 foram lidos por completo e o volume de artigos resultantes neste passo foram utilizados para compor a revisão da literatura e apoiar nas respostas das questões de pesquisa (QP).

Figura 1

Passos da estratégia de

seleção de trabalhos

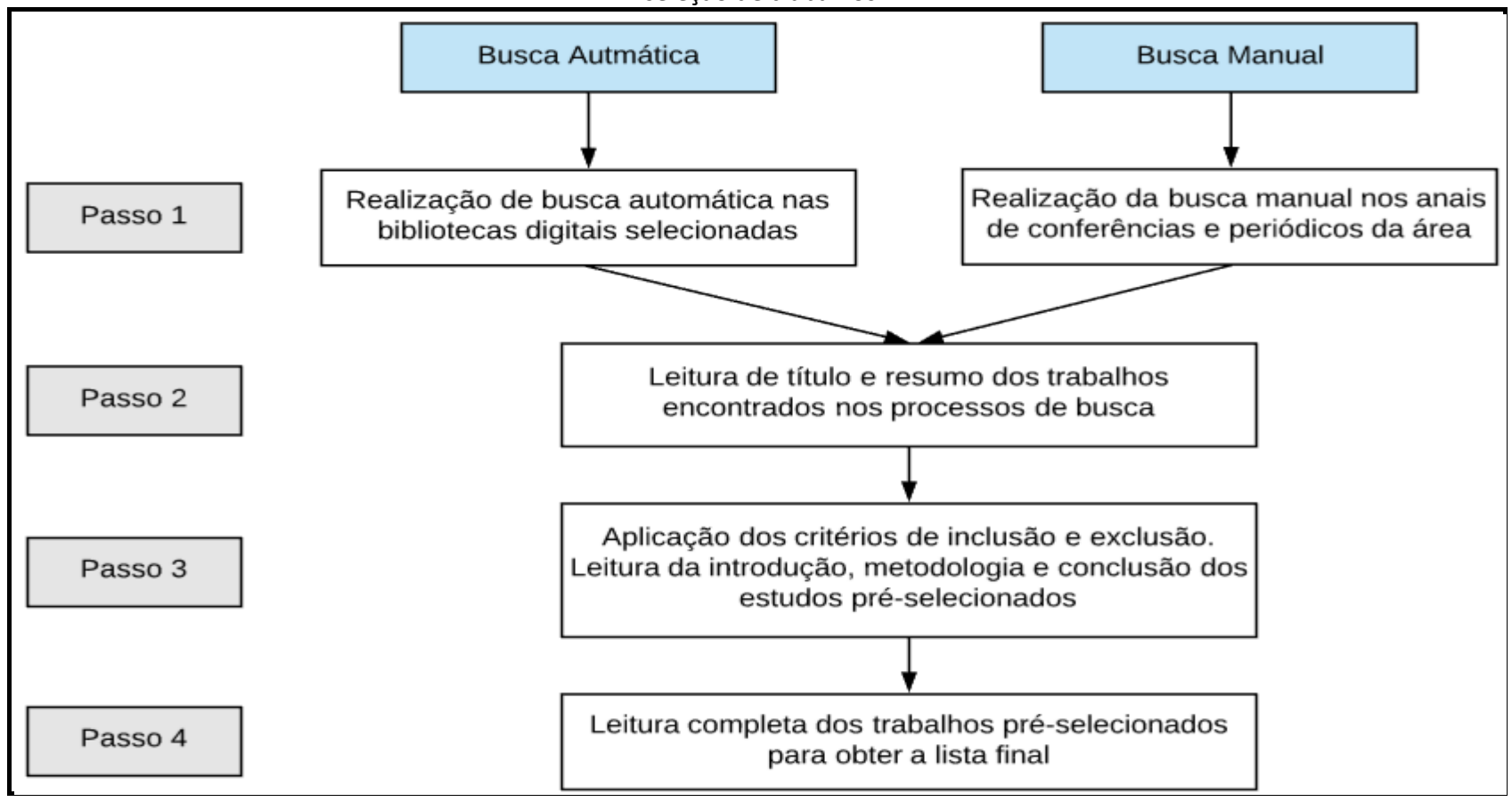




\subsection{Seleção de Estudos Primários}

Iniciou-se o processo de seleção automática dos estudos primários através da execução da string de busca nas bases digitais. Como cada uma das bases possui critérios específicos de busca, seja pelos operadores permitidos ou pelos recursos disponibilizados, algumas adequações na string de busca precisaram ser feitas. A busca automática nas 3 bases definidas resultou num total de 1008 artigos, sendo 487 ou 48,3\% dos artigos na Digital library ACM e 377 ou 37,4\% dos artigos identificados na Digital library IEEE Xplore e 144 ou 14,3\% dos artigos localizados na Eric (Institute of Educaction Sciences).

A busca manual realizada nos Anais das Conferências e Periódicos determinados na Tabela 2 foi realizada através de combinações variadas da string de busca definida no protocolo. Essa variação da string se fez necessária pois as bases das Conferências e Periódicos possuem um volume menor de publicações e com a string completa muitas vezes não era identificado nenhum trabalho. A busca manual resultou em um total de 42 artigos. Com isso, a seleção final de artigos para o passo 1, foi de 1050 artigos.

Tabela 2

Endereço de periódicos e conferências

utilizados na busca manual

\begin{tabular}{|c|l|}
\hline Sigla & Conferência / Periódico \\
\hline IJAIE & International Journal of Artificial Intelligence in Education \\
\hline ALT & Association for Learning Thecnology \\
\hline ATPE & Technology, Pedagogy and Education Association \\
\hline AJET & Australasian Journal of Educational Technology \\
\hline JECR & Journal of Educational Computing Research \\
\hline JEP & Educational Psychologist Journal \\
\hline JCAL & Journal of Computer Assisted Learning \\
\hline
\end{tabular}

A evolução da seleção destes na revisão sistemática de literatura pode ser verificada de forma sintética na Tabela 3. Com a finalização do passo 3 da estratégia de busca, que consistiu na leitura da introdução, metodologia e conclusão dos trabalhos, foram pré selecionados 14 artigos e já agrupados para leitura completa (passo 4) visando responder as questões de pesquisa (QP).

Tabela 3

Evolução da Estratégia de Seleção

\begin{tabular}{|c|c|c|c|c|}
\hline Estratégia & Passo 1 & Passo 2 & Passo 3 & Passo 4 \\
\hline Busca Automática & 1008 & 151 & 65 & 11 \\
\hline Busca Manual & 42 & 22 & 13 & 3 \\
\hline Total Selecionado & 1050 & 173 & 78 & 14 \\
\hline
\end{tabular}

Fonte: Autor

\section{Resultados}

A lista de artigos candidatos foi refinada através da leitura de todos os artigos e da aplicação dos critérios de exclusão. A extração dos dados ocorreu em todos os 14 artigos selecionados no último passo da estratégia definida no protocolo desta pesquisa.

A partir da leitura completa desses artigos apresentados na Tabela 4 foi possível responder as questões de pesquisa. Na Tabela 4, a coluna ID apresenta o código identificador do artigo selecionado para a pesquisa. A coluna Autor, Ref, exibe o nome do primeiro autor do artigo selecionado, bem como a referência do artigo. A 
coluna Ano refere-se ao ano de publicação da pesquisa. A coluna Descrição apresenta uma pequena explicação do contexto do trabalho. A coluna QP mostra em qual/quais questões de pesquisa desta revisão sistemática da literatura foram utilizados o artigo.

Tabela 4

Breve descrição dos artigos selecionados

\begin{tabular}{|c|c|c|c|c|}
\hline Id & Autor-Ref & Descrição & Ano & QP \\
\hline E1 & $\begin{array}{l}\text { Thammathip, } \\
\text { P. et al., }[X]\end{array}$ & $\begin{array}{l}\text { Analisa e explora técnicas para aprimorar a colaboração remota de realidade } \\
\text { mista (RM) em termos de comunicação e interação com ambientes } \\
\text { tecnológicos. }\end{array}$ & 2017 & 1 \\
\hline E2 & $\begin{array}{l}\text { Kim J. et al., } \\
\qquad[\mathrm{X}]\end{array}$ & $\begin{array}{c}\text { Cria ambiente de aprendizagem para linguagem de programação para o apoio } \\
\text { ao aprendizado usando técnicas para aprimorar a colaboração remota de } \\
\text { realidade mista (RM). }\end{array}$ & 2019 & 1 \\
\hline E3 & $\begin{array}{l}\text { Ali, A. et al., } \\
{[\mathrm{X}]}\end{array}$ & $\begin{array}{l}\text { Mapeia estudos de ambientes educacionais colaborativos que incorporam } \\
\text { tecnologias de realidade mista (RM). }\end{array}$ & 2019 & 1 \\
\hline E4 & $\begin{array}{l}\text { Andone, D. et } \\
\text { al., }[X]\end{array}$ & $\begin{array}{l}\text { Apresenta um estudo colaborativo de aprendizagem que utiliza realidade virtual } \\
\text { (RV)para melhorar o aprendizado do aluno na área de tecnologia. }\end{array}$ & 2018 & 1,2 \\
\hline E5 & $\begin{array}{l}\text { Campbell, A et } \\
\text { al., [X] }\end{array}$ & $\begin{array}{c}\text { Apresenta um ambiente de aprendizagem de realidade de mista multiusuário } \\
\text { ente professores e alunos. }\end{array}$ & 2016 & 1,2 \\
\hline E6 & $\begin{array}{l}\text { Erik Kucera, } \\
\qquad[\mathrm{X}]\end{array}$ & $\begin{array}{l}\text { Apresenta proposta para melhorar a qualidade do ensino para graduados e } \\
\text { especialistas em programas de estudos } \\
\text { técnicos de mecatrônica e áreas relacionadas de automação usando realidade } \\
\text { mista (RM). }\end{array}$ & 2018 & 1,2 \\
\hline E7 & $\begin{array}{l}\text { Ralph, D. et } \\
\quad \text { al., }[\mathrm{X}]\end{array}$ & $\begin{array}{l}\text { Apresenta um levantamento das abordagens usadas para medir a aprendizagem } \\
\text { (para combinar métricas de presença, imersão) e estabelecer a eficácia de uma } \\
\text { experiência de aprendizado de realidade virtual (RV) na educação. }\end{array}$ & 2017 & $1,2,3$ \\
\hline E8 & $\begin{array}{l}\text { Yannier, N. et } \\
\text { al., [X] }\end{array}$ & $\begin{array}{c}\text { Investiga se a aprendizagem ativa é melhor quando implementada por um } \\
\text { sistema de inteligência artificial (IA) por meio da realidade mista (RM) para } \\
\text { apoiar o ensino STEM para crianças. }\end{array}$ & 2020 & 1,2 \\
\hline E9 & $\begin{array}{l}\text { Vasilevski , N.; } \\
\text { Birt, J. [X] }\end{array}$ & $\begin{array}{l}\text { Apresenta estudo e avalia as experiências dos estudantes de engenharia } \\
\text { proporcionadas pelo aprendizado usando uma combinação de realidade virtual } \\
\text { e aumentada. }\end{array}$ & 2020 & 2 \\
\hline E10 & $\begin{array}{l}\text { Maas M., } \\
\text { Hughes J., }[\mathrm{X}]\end{array}$ & $\begin{array}{l}\text { Apresenta revisão da literatura existente sobre o uso de tecnologias de } \\
\text { realidade virtual (RV), aumentada (RA) e mista (RM) no K - } 12 \text { (ensino } \\
\text { educacional nos EUA que está no intervalo entre o primário e o secundário). }\end{array}$ & 2018 & 1,2 \\
\hline E11 & Cheng H., $[\mathrm{X}]$ & $\begin{array}{c}\text { Apresenta estudo que investiga s relações entre a carga cognitiva, a motivação e } \\
\text { as atitudes percebidas pelos alunos quando utilizam um ambiente tecnológico } \\
\text { de realidade aumentada (RA). }\end{array}$ & 2018 & $1,2,3$ \\
\hline E12 & Beck, D., & $\begin{array}{l}\text { Apresenta pesquisas sobre as potencialidades, procedimentos e desafios de } \\
\text { ambientes de aprendizagem imersivos com o foco no uso ideal de mundos e } \\
\text { ambientes imersivos para fins educacionais por meio de da aplicação da } \\
\text { realidade mista (RM). }\end{array}$ & 2017 & 1,2 \\
\hline E13 & $\begin{array}{l}\text { Anmarkrud, } \mathrm{O} . \\
\text { et al., [X] }\end{array}$ & $\begin{array}{l}\text { Apresenta pesquisa sobre aprendizagem multimídia que usa a teoria da carga } \\
\text { cognitiva como o principal arcabouço teórico. }\end{array}$ & 2020 & 3 \\
\hline E14 & $\begin{array}{l}\text { Dan A., Reiter } \\
\text { M. [X] }\end{array}$ & $\begin{array}{l}\text { Apresenta estudo comparativo dos ambientes de aprendizagem 2D e 3D para } \\
\text { obter uma redução na carga cognitiva, aprimoramento da aprendizagem e um } \\
\text { ajuste adequado às necessidades e capacidades do aluno. }\end{array}$ & 2020 & 2,3 \\
\hline
\end{tabular}

Fonte: Autor 


\subsection{QP.1. Como são incorporados os ambientes de aprendizagem imersivos a qualquer nível educacional e como são aplicados os tipos de realidades diferentes (realidade aumentada, realidade virtual, realidade mista)?}

O estudo (Piumsomboon, et al., 2017), propõe a construção de um ambiente de RM para aprimorar o uso da interface e comunicação entre uma comunidade de alunos e professores para uma colaboração remota utilizando o dispositivo de RM, o HoloLens. Permite que as pessoas distantes se sintam no mesmo espaço físico. Para criar uma experiência colaborativa contínua, o sistema criado fornece três métodos de entrada comuns na plataforma de RM: movimento da cabeça do usuário (OHMD), rastreamento do olhar do usuário e controle de gestos com as mãos. $O$ ambiente tem como objetivo melhorar a interação e comunicação por meio da interface. O Covar permite compartilhar conteúdos 3D virtual e tem como destaque a possibilidade de reconstruir um objeto 3D em tempo real para colaborar com tarefas espaciais no espaço de aprendizagem compartilhado, aprimorar a comunicação, incluindo dicas de interface, e também técnicas para aprimorar a interação com a interação natural colaborativa e o dimensionamento do corpo do usuário. Após o estudo exploratório, encontrou-se evidências que sustentam os benefícios destas melhorias e dicas para aprimorar a colaboração e testar o sistema com uma tarefa específica do mundo real.

Na pesquisa (Maas \& Hughes, 2020), fornece uma revisão da literatura sobre o uso de tecnologias de RV, RA e RM em ambientes educacionais conhecido como $k-12$ - ensino educacional nos EUA (Estados Unidos da América), que está no intervalo que ocorre entre o primário e o secundário. Dos 29 estudos incluídos, 24 analisaram a RA, 3 analisaram a RM e dois analisaram a RV. Os principias estudos apresentados, concentraramse em assuntos STEM (disciplinas de ciência, tecnologia, engenharia e matemática). Os estudos de matemática incluíam álgebra e geometria (Estapa \& Nadolny, 2015), formas geométricas e matemática (Laine, Nygren, Dirin, \& Suk, 2016), artes da linguagem e cientifica e alfabetização (Dunleavy, C., \& Mitchell, 2009). Os tópicos científicos incluíram eletrostática com cargas pontuais e eletricidade estática (Echeverria, Amestica, F., Barrios, \& Leclerc, 2012), habilidades de argumentação em ciências ambientais, educação ambiental e viagens de campo (Kamarainen, et al., 2013), ciências da escola primaria (Kerawalla, Luckin, Seljeflot, \& Woolard, 2006)), ciclos de agua (Furió, Juan, Seguí, \& Vivó, 2015) , bactérias (Drachsler, Hung, Chen, \& Huang, 2017) condutividade elétrica (Yoon, Elinich, Wang,, Steinmeier, \& Tucker, 2012), eletromagnetismo (Ibáñez, Á., Villarán, \& C, 2014). Os estudos de RV foram realizados pelo tipo de ambiente desktop, na área de ciências e biologia (Lee \& Wong, 2014) e o uso da RV imersivo com interface háptica (interface tátil em que um sistema fornece respostas ao usuário) para a aprendizagem da área de física, que possibilita aos usuários dos ambientes imersivos sentirem os objetos virtuais com uma qualidade muito superior aos mecanismos vibratórios dos controles atuais, (Civelek, Ucar, Ustunel, \& Aydin, 2014).

Os estudos de RM revisados em (Maas \& Hughes, 2020) envolveram o uso de um ambiente virtual desktop para aprender sobre a evolução geológica (Birchfield \& Megowan-Romanowicz, 2009) usando uma tecnologia de projeção holográfica para instruir sobre o espaço sideral (Lindgren, Tscholl, Wang, \& Johnson, 2016).

Aplicou-se uma metodologia de testes com os alunos em grupos separados antes e após o uso dos ambientes de aprendizagem por meio de questionários e concluiu-se estatisticamente sobre o desempenho de aprendizagem: a)os alunos responderam a mais perguntas corretamente após o uso de RA; b)ajudou a esclarecer e simplificar os materiais de aprendizagem (Chen, Chou, \& Huang, 2016); c)descobriu-se que não havia diferença na retenção de aprendizagem do material entre o uso de um livro gráfico de RA e um livro de figuras e a interação física ao ensinar aos alunos; d)um estudo do ensino médio mostrou que os alunos superaram as alunas a um nível estatisticamente significativo na utilização de um ambiente de RV, no entanto, o mesmo estudo determinou que não havia importância significativa na forma de aprender entre o ambiente de RA e o ambiente que não possuía RA, (Chang, Lee, Wang, \& Chen, 2010); e) por meio de dados observacionais qualitativos, alunos do ensino 
fundamental que lutavam com a leitura tradicional também lutavam com a interação RA (Echeverria, Amestica, F., Barrios, \& Leclerc, 2012); f)alunos que aprendem conceitos abstratos de física em um ambiente de RV obtiveram maiores ganhos de aprendizagem do que os alunos de uma aula regular tradicional (Civelek, Ucar, Ustunel, \& Aydin, 2014); g)um grande número de estudantes do ensino médio sentiu que o uso de uma simulação de RA háptica (interface tátil em que um sistema fornece respostas ao usuário) os ajudaria significativamente a aprender melhor conceitos abstratos de física, como atração gravitacional (Civelek, Ucar, Ustunel, \& Aydin, 2014).

No estudo de (Ali, Dafoulas, \& Augusto, 2017), foi realizada uma revisão sistemática com foco no impacto das tecnologias de RM na aprendizagem colaborativa com atividades educacionais. Os resultados sugerem que vários estudos se concentraram em observar as formas de impacto na perspectiva da aprendizagem e no processo de aquisição de conhecimentos ou habilidades (Kitchenham, 2019), (Dünse, R., \& Billinghurst, 2008), (Callaghan, et al., 2008), (Microsoft, 2019), (Zou, et al., 2017).Também foi demonstrado o resultado do estudo proposto para melhorar o uso de uma interface de RA para a aprendizagem colaborativa, apontando consequências positivas na relação de ensino. Outro resultado mostrado nesta revisão sistemática que usou ambiente totalmente imersivo como o headsets $\mathrm{OHMD}$, onde os usuários foram capazes de visualizar e manipular representações em 3D de componentes de hardware de computadores. Foi exposto evidências de que os headsets OHMD podem ser usados de várias maneiras para apoiar atividades de aprendizagem, como por exemplo prover retorno aos alunos sobre as atividades realizadas; contribuir com o suporte de tutor para uma variedade de tarefas; observação por pares e também avaliação de processos de aprendizagem (Dillenbourg, 1999), (Google, 2017). Ainda nesse estudo, foi abordado o conceito que discutia oportunidades para jogos de RM e cenários relacionados para a aprendizagem, e mostrou várias questões e desafios educacionais a serem tratados especificamente ao vincular RA e virtualidade aumentada.

Projetos para criação de ambientes imersivos de aprendizagem estão levando em consideração todos as tecnologias atuais para a sua estrutura e de forma hibrida, combinando a sala de aula tradicional e os ambientes imersivos de aprendizagem on-line. Um exemplo de ambiente inovador de projeto (Yannier, Hudson, \& Koedinger, 2020), que apostou no uso dos benéficos da Inteligência Artificial (IA) a seu favor, programando um algoritmo de visão computacional. O projeto foi criado para apoiar as crianças na aprendizagem de ciência e contribuiu para melhorar o entendimento que as crianças possuem quando submetidas a uma orientação específica na aprendizagem tecnológica. Os resultados apresentados demonstram que o ambiente de RM inovador que foi utilizado colabora com as crianças que aprendem com uma orientação adaptativa proporcionada pela IA, alcançam maior entendimento sobre o conteúdo e os resultados da metodologia dos testes demonstraram que as crianças ganham aprendizado na proporção de vezes mais do que as crianças que aprendem apenas utilizando a construção prática sem a orientação especifica da IA. Esses resultados sugerem que os sistemas de RM com IA podem ser mais eficazes quando formas de orientação interativa são adicionadas para facilitar a aprendizagem ativa e a compreensão dos entendimentos científicos fundamentais, em vez de focar somente e exclusivamente na exploração prática sem ter o retorno do processo de aprendizagem.

Outro exemplo é o estudo que tem como objetivo criar um projeto de espaços de RM, nos quais professores e alunos podem não apenas estar virtualmente presentes em qualquer lugar do mundo, mas também estar fisicamente presentes (Campbell, Santiago, Hoo, \& Mangina, 2016). Esse ambiente educacional futuro poderá oferecer ao seu usuário interfaces de interação diferentes, desde de uma interface de ambiente 3D desktop a uma interface imersiva 3D completa usando headsets OHMD de RV, sendo possível também a utilização de vídeo panorâmicos 360 graus e hologramas com espaço compartilhado que permitirá que professores holográficos 2D sejam uma entidade virtual na sala de aula do mundo real por meio de avatar. 
Em relação a utilização de hologramas, eles podem ser projetados em um ambiente real, permitindo interações com gestos e vozes que devem ser programados pelos seus desenvolvedores para realizar ações específicas de um projeto (Microsoft, 2019). Um estudo que desenvolveu uma ferramenta com esse conceito que contém cenas com gráficos 3D interativos para a RM com o objetivo de apoiar o ensino técnicos e de bacharelados que envolvem mecatrônica e outras áreas da engenharia (Kucera, Haffner, \& Leskovský, 2018). As imagens 1 e 2 mostram a utilização de hologramas para a aprendizagem de conteúdo por meio do Microsoft HoloLens. $O$ estudo mostra claramente a possibilidade da fusão do mundo real e virtual para produzir novos ambientes e visualização onde objetos físicos e digitas coexistem e interagem em tempo real, sobrepondo-se entre eles e são capazes de reagir um ao outro e obter uma motivação maior para construir a aprendizagem.

Imagens 1 e 2

Microsoft HoloLens - aplicativo de realidade mista (RM)

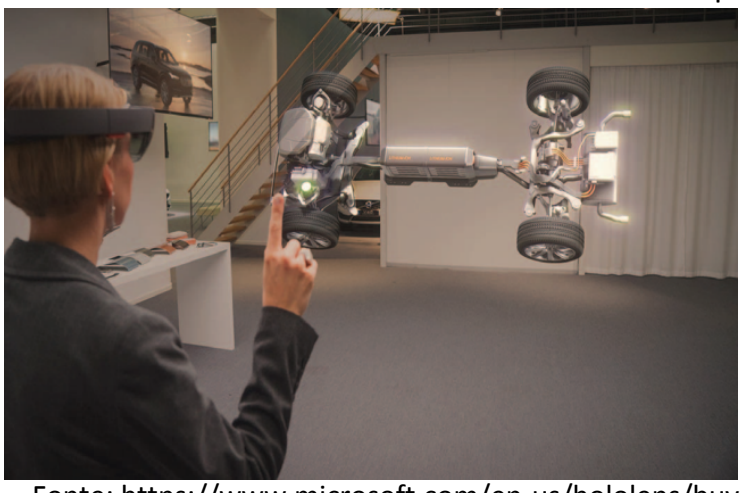

Fonte: https://www.microsoft.com/en-us/hololens/buy

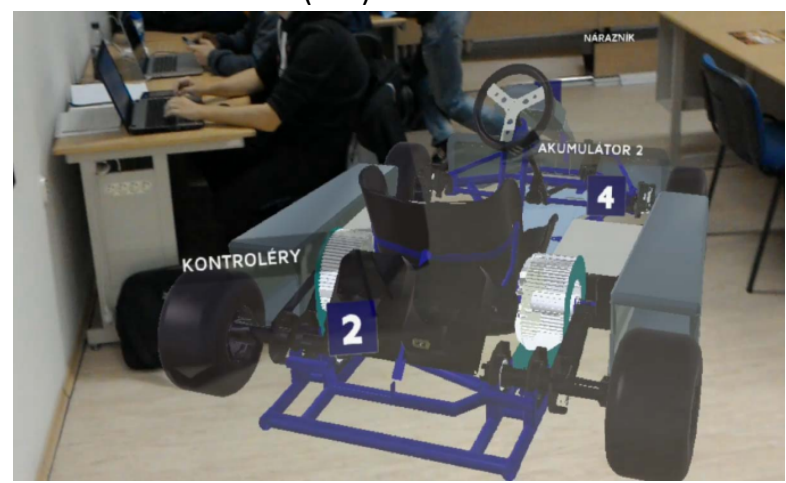

Fonte: https://www.microsoft.com/en-us/hololens/buy

\subsection{QP2. Como as teorias de aprendizagem são utilizadas e relacionados com os ambientes de aprendizagem?}

A mediação pedagógica é importante nesse contexto de ambientes de aprendizagem tecnológico. Professores assumem a função de mediar a relação da aprendizagem do aluno com a tecnologia. Propondo roteiros e atividades de desenvolvimento educacionais que podem ser fundamentados nas teorias que aprendizagem.

Algumas teorias de aprendizagem são utilizadas nos ambientes para corroborar com evidências de melhoria na relação de ensino/aprendizagem. Muitos estudos correlacionam algumas dessas teorias para fazer parte do arcabouço teórico e justificar a forma de como a pedagogia ou heutagogia poderá ser utilizada na construção da aprendizagem utilizando-se dos ambientes tecnológicos (Ali, Dafoulas, \& Augusto, 2017), (Campbell, Santiago, Hoo, \& Mangina, 2016), (Ralph, et al., 2017), (Yannier, Hudson, \& Koedinger, 2020), (Beck, 2019).

Um dos conceitos utilizado é o de zona de desenvolvimento proximal de Vygotsky (Vygostky, 1987), que define a distância entre o nível de desenvolvimento real, determinado pela capacidade de resolver um problema sem ajuda e o nível de desenvolvimento potencial determinado através de resolução de um problema sobre a orientação de um adulto ou em colaboração com algo, por exemplo uma criança mais velha. A RV, nesse aspecto, pode se tornar uma ferramenta adequada para atender os requisitos da zona de desenvolvimento proximal. Ela pode ser usada como uma ferramenta de aprendizagem que pode preencher a lacuna de conhecimento, segundo demonstra o estudo de (Campbell, Santiago, Hoo, \& Mangina, 2016).

No estudo (Ralph, et al., 2017), utiliza a teoria de aprendizagem do construtivismo que em geral, estabelece como as pessoas aprendem usando conhecimentos e experiências anteriores para construir o entendimento através de experiências colaborativas ativas. Os professores incentivaram os alunos a relacionar, experimentar, aplicar, cooperar e transferir conhecimentos e entendimentos. Nesse estudo o ambiente de RV pode fornecer a 
professores e alunos a capacidade de estabelecer condições ideais para a aprendizagem, oferecendo oportunidades para a exposição dos alunos a problemas complexos, abordados em um ambiente colaborativo e que exigem engajamento ativo. $O$ construtivismo pode fundamentar a medição do conhecimento do conteúdo e da aprendizagem utilizando-se da RV.

O estudo (Yannier, Hudson, \& Koedinger, 2020), utilizou uma análise randomizada e controlada que investiga se a aprendizagem ativa é melhor quando implementada como uma prática deliberada guiada, como a teoria construtivista de aprendizagem, ou como uma combinação de ambos. A orientação reativa e automatizada é possibilitada por um algoritmo especializado de visão computacional da IA, desenvolvido para rastrear o que as crianças estão fazendo no ambiente físico enquanto realizam experimentos e descobertas com objetos físicos. Os resultados deste estudo apoiam a prática deliberada e indicam que ter alguma descoberta guiada com base em um mecanismo de aprendizagem eficaz, como auto explicação, casos contrastantes e feedback interativo personalizado, produz uma aprendizagem mais robusto comparado à construção exploratória. Nesse estudo a prática deliberada enfatiza a estrutura planejada, particularmente na forma de design de tarefas específicas e de feedback explicativo imediato. O construtivismo enfatiza a criação de saídas externalizadas, o uso de tarefas autênticas ou lúdicas e o alerta contra muita instrução.

Alguns estudos selecionados ficaram em uma área cinzenta de classificação. Eles demostram resultados de aprendizagens, mas não citam embasamentos nas teorias de aprendizagem.

\subsection{QP3. Como é a relação entre o ambiente aprendizagem tecnológico e o ensino por meio da teoria da carga cognitiva?}

Há estudos consistentes que mostram a hipótese da relação entre carga cognitiva e aprendizagem multimidia (Anmarkrud, Andresen, \& Bråten, 2019). A teoria da carga cognitiva tem sido muito importante em pesquisas sobre os potenciais benefícios do uso de ambientes de aprendizagem imersivos tecnológicos e na construção de conexões entre ciência cognitiva e a prática instrucional e ao mesmo tempo tem sido objeto de crítica no que diz respeito a medição. O estudo de (Anmarkrud, Andresen, \& Bråten, 2019) fez uma revisão da pesquisa sobre aprendizagem multimidia pela perspectiva da teoria da carga cognitiva. Identificar possíveis problemas com a conceituação e medição da memória de trabalho e sobre a medição da carga cognitiva foi examinado uma amostra de estudos recentes de aprendizagem multimidia enquadrados pela teoria da carga cognitiva. Mostrou que 32 de 73 estudos avaliados são consistentes com a hipótese da relação entre carga cognitiva e aprendizagem multimidia, o que implica que bons projetos de design instrucional levam a níveis reduzidos de carga cognitiva, bem como ao aprimoramento da aprendizagem multimidia devido a demandas de processamento reduzidas na memória de trabalho. Porém nos outros estudos pesquisados, o design instrucional testado mostra melhora na aprendizagem sem redução da carga cognitiva ou redução de carga cognitiva sem efeito de aprendizagem Uma da razões que pode justificar essa relação inconsistente que foi mostrada é o uso de medidas subjetivas de carga cognitiva caracterizado por medidas gerais, incluindo muito poucos itens, falta de informações psicométricas, falta de combinação com outros tipos de medidas de carga cognitiva e operacionalização inconsistente, além de falta de descrições claras da procedimentos utilizados para medir a carga cognitiva. Isso é problemático e são necessárias medidas sensíveis da carga cognitiva para entender os mecanismos cognitivos subjacentes aos efeitos ou a falta deles nos projetos instrucionais (Anmarkrud, Andresen, \& Bråten, 2019).

O estudo (Cheng K.-H. , 2017) por meio de uma pesquisa quantitativa explorou as relações entre a percepção da carga cognitiva, a motivação e a percepção das atitudes. O estudo criou um livro de RA para ser lido por estudantes. A leitura possui auxílio de RA que combina as vantagens do livro físico com o conteúdo digital, incluindo possiblidades intuitivas de interação. Especificamente esses tipos de sistemas de contexto tecnológico utilizando RA, são capazes de preencher um espaço entre objetos físicos e virtuais com tendência de melhorar mais a compreensão dos leitores sobre o conteúdo, especialmente em termos de conceitos abstratos que não 
podem ser bem compreendidos. Com base na análise de dados do estudo, a carga cognitiva, a motivação e as atitudes percebidas pelos estudantes ao se envolverem na leitura do livro de RA foram mostradas. Quanto à carga cognitiva, não foram encontradas diferenças significativas entre a percepção do esforço mental e a carga mental dos alunos, e a percepção da carga cognitiva pelos alunos na atividade de aprendizagem de realidade mental foi geralmente de baixo nível, isto é, os estudantes não precisaram de carga mental, nem investiram esforço mental, muita capacidade cognitiva no processamento das informações do livro de RA. Esse resultado corresponde a estudos anteriores que relataram uma diminuição da carga cognitiva nos ambientes de aprendizagem de RA (Klatzky, Wu, Shelton, \& Stetten, 2008).

A pesquisa (Dan \& Reiner, 2017), comparou a aprendizagem em dois cenários de aprendizado virtual - em telas $2 \mathrm{D}$ e com um cenário 3D idêntico. Os participantes assistiram a um vídeo 2D ou 3D de um instrutor demonstrando uma tarefa de estrutura dobrável de caixa de papel origami. Foram comparados os resultados dos testes dobráveis dos participantes e aplicado questionários de avaliação dos cenários de ensino que calculou seu índice de carga cognitiva (CLI - cognitive load index) com base nas medidas do eletroencefalograma durante os períodos de observação. Procurando assim, a relação entre carga cognitiva, aprendizagem e desempenho em cada uma das configurações de aprendizagem. O estudo mostrou evidencias que os esforços mentais dos participantes durante a observação no plano 2D foi muito maior em comparação com os esforços mentais necessários durante o 3D no mesmo período, isto é, as tecnologias imersivas 3D são vantajosas para melhorar a aprendizagem e reduzir a carga cognitiva. Os resultados sugerem também novas descobertas sobre os benefícios de utilização de uma avatar humano realista do projetado em 3D em comparação com um vídeo 2D. Cargas cognitivas externas associadas a fatores como excesso de informação, tarefas complexas, interfaces artificiais não amigáveis e ambiente sensorial barulhento interferem nos processos de aprendizagem/ memorização (Hollender, Hofmann, Deneke, \& Schmitz, 2010).

\section{Conclusão}

É perceptível que a tecnologia tem sido empregada na área educacional com bastante pluralidade na perspectiva de áreas de conhecimento, metodologias, abordagens pedagógicas e principalmente resultados obtidos referente a um potencial ganho de aprendizagem. É desafiador responder prontamente algumas indagações pontuais que envolvem tecnologia e educação. Novos rumos na educação tecnológica são empregados, os espaços de aprendizagem que se restringem as salas de aulas, atualmente, são oferecidos por meio de ambientes tecnológicos sendo possível interagir e participar deste novo espaço de ambiente de aprendizagem a qualquer momento sem restrição de tempo e espaço.

Os ambientes virtuais de aprendizagem estão evoluindo significativamente para oferecem condições de interação síncrona e assíncrona entre seus alunos e professores, que definem os próprios caminhos para o acesso à informação, podendo garantir uma aprendizagem personalizada e no seu próprio ritmo. Eles trouxeram novas perspectivas para educação. Com base na maioria dos estudos que integram tecnologias no espectro da RM em diferentes ambientes e áreas de conhecimentos específicos (Piumsomboon, et al., 2017), (Kim, et al., 2019), (Ali, Dafoulas, \& Augusto, 2017), (Andone, Frydenberg, Vert, \& Vasiu, 2018), (Campbell, Santiago, Hoo, \& Mangina, 2016), (Kucera, Haffner, \& Leskovský, 2018), (Maas \& Hughes, 2020), (Cheng K.-H. , 2017), observa-se impactos positivos com maior relevância de que aspectos negativos da incorporação tecnológica na educação.

Ainda que haja alguns tipos de limitações, as utilização da tecnologias de ambientes de RM incorporadas às atividades educacionais estão criando possibilidades de desenvolver experiências significativas de aprendizagem por meio de novas formas de interação, como deteç̧ão de gestos e emoções, novas formas de comunicação por meio de melhorias interfaces instrucionais e o ambiente que pode ser colaborativo para proporcionar a troca de experiencias e discussões (Ali, Dafoulas, \& Augusto, 2017), (Vasilevski \& Birt, 2020), (Beck, 2019). 
Muitos estudos se direcionaram para aplicação da tecnologia mista para áreas e níveis educacionais próprios, como o da mecatrônica, aplicado no ensino médio técnico e graduação de engenharias (Kucera, Haffner, \& Leskovský, 2018). Foi utilizado a tecnologia da holografia no ambiente para demonstrar evidências positivas que impactaram diretamente na aprendizagem.

Por outro lado, as teorias de aprendizagem podem afetar o ensino e podem influir na escolha de uma solução tecnológica que seja apropriada para professores e alunos. Por isso, é importante identificar características educacionais especificas de uma mídia. Elas forneceram uma base para sugerir e avaliar diferentes formas de ensino e para atender às diversas necessidades para se aprender na sociedade em redes.

Embora, as teorias de behaviorismo, cognitivismo e construtivismo são citadas em muitos estudos que em ambientes imersivos de aprendizagem (Ali, Dafoulas, \& Augusto, 2017), (Campbell, Santiago, Hoo, \& Mangina, 2016), (Ralph, et al., 2017), (Yannier, Hudson, \& Koedinger, 2020), (Beck, 2019), o construtivismo tem sido utilizado com forte ênfase na área educacional tecnológica. Aprendizagem colaborativa e aprendizagem imersiva on-line, e comunidades de práticas são os principais métodos construtivistas utilizados na aprendizagem tecnológica.

Vários estudos apontam para um potencial aprimoramento da aprendizagem devido a utilização de AVA, as relações entre carga mental e aprendizagem precisão avançar com mais exatidão. Fazer a correlação de ganho de aprendizagem no processo de cognição humano por meio da utilização da tecnologia tem se demonstrado um desafio. A teoria da carga cognitiva tenta explicar como a carga de processamento de informações, que surge durante o ensino de novas informações, afeta o processamento do conhecimento dos alunos e a estruturação do conhecimento em termo de memória (Sweller, Merriënboer, \& Paas, 2019).

Medir a carga cognitiva de maneira confiável, válida e sensível é uma tarefa complexa, e alguns pesquisadores da teoria da carga cognitiva investiram muito esforço no desenvolvimento de medidas mais objetivas da carga cognitiva e na comparação com medidas subjetivas (Korbach, Brunken, \& Park, 2017), (Korbach, Brunken, \& Park, 2018), (Park, Korbach, \& Brunken, 2015).

O desenvolvimento de tecnologias mais sofisticadas levou a um crescente interesse em medidas fisiológicas da carga cognitiva, e começaram a surgir evidências de outras técnicas têm mérito considerável, como a resposta papilar cognitiva, ressonância magnética funcional e eletroencefalografia (Pass, Ayres, \& Pachman, 2008), (Sweller, Ayres, \& Kalyuga, 2011).

Também há dentro do contexto de medição da aprendizagem multimídia, o uso crescente da metodologia de rastreamento ocular para examinar as mudanças no processamento cognitivo, e tem sido argumentado que maior tempo de fixação ocular e maior tamanho da pupila estão relacionados ao aumento da carga cognitiva (Chuang \& Liu, 2012), (Underwood, Jebbet, \& Roberts, 2004), (Gog \& Jarodzka, 2013). Os ambientes de aprendizagem que levam a muita imersão e, consequentemente, a um forte senso de presença, tem tendências de diminuir a carga cognitiva medida e resultar em uma progressão da aprendizagem (Dan \& Reiner, 2017).

Os alunos com menos carga cognitiva podem ter uma motivação mais forte e atitudes mais positivas ao se envolverem em atividades com ambientes tecnológicos imersivos, por exemplo a leitura de livros utilizando a tecnologia de RA. No entanto, de acordo a percepção da carga cognitiva pelos estudantes não pode prever diretamente suas atitudes em relação às intenções comportamentais, isto é, embora os alunos leiam o livro de RA com menos esforços mentais, as percepções de carga cognitiva de baixo nível não podem ser garantidas de forma positiva para o aluno aprender no futuro (Cheng K.-H. , 2017). Portanto, usar outros tipos de medidas fisiológicas nos ambientes de aprendizagem virtual imersivos faz-se necessário para explorar o potencial ganho de aprendizagem que a tecnologia pode oferecer com confiabilidade. 


\section{Referência Bibliográfica}

Ali, A. A., Dafoulas, G. A., \& Augusto, J. C. (2017, October 15). Collaborative Educational Environments Incorporating Mixed Reality Technologies: A Systematic Mapping Study. Transactions on Learning Technologies, pp. 321 - 332.

Andone, D., Frydenberg, M., Vert, S., \& Vasiu, R. (2018, June 9-13). Open Virtual Reality Project to Improve Students' Skills. 18th International Conference on Advanced Learning Technologies, pp. 6-10.

Anmarkrud, Ø., Andresen, A., \& Bråten, I. (2019, February 11). Cognitive Load and Working Memory in Multimedia Learning: Conceptual and Measurement Issues. EDUCATIONAL PSYCHOLOGIST, pp. 1-23.

Beck, D. (2019, June 25). Special Issue: Augmented and Virtual Reality in Education: Immersive Learning Research. Journal of Educational Computing, pp. 1619-1625.

Birchfield, D., \& M.-R. C. (2009, April 4). Earth science learning in SMALLAB: A design experimente for mixed reality. International Journal of Computer-Supported Collaborative Learnning, pp. 403-421.

Callaghan, V., Garnder, M., Horan, B., Scott, J., Shen, L., \& M. Wang. (2008). A mixed reality teaching and learning environment. ConfHybrid Leraning and Education, 54-65.

Campbell, A. G., Santiago, K., Hoo, D., \& Mangina, E. (2016, December 6-7). Future Mixed Reality Educational Spaces. Future Mixed Reality Educational Spaces, pp. 1088-1093.

Chang, C., Lee, C., Wang, J., \& Chen. (2010). Improving the authentic learning experience by integrating robots into the mixed-rality environment. Computers \& Education, pp. 1572-1578.

Chen, C., \& Tsai, Y. (2012). Interactive augmented reality system to improve the teaching of the library in rpimary schools. Computer and Education Journal , pp. 638-652.

Chen, C., Chou, Y., \& Huang, C. (2016, April 25). An augmented'reality-based concept map to support learning for science. THe Asia-Pacif Education Research, pp. 567-578.

Cheng, K., \& Tsai, C. (2016). The interaction of child-parent shared reading whith an augmented reality (AR) picture book and parentes conceptions of AR learning: Child-parent augmented reality book reading. British Journal of Education Technology, pp. 203-222.

Cheng, K.-H. (2017). Reading an augmented reality book: An exploration of learners cognitive load, motivation, and attitudes. Australasian Journal of Educational Technology, pp. 53-69.

Chiang, T., Yang, S., \& G, H. (2014). Students online interactive patterns in augmented reality'based inquiry activities. Computers \& Education, pp. 97-108.

Chuang, H., \& Liu, H. (2012). Effects of different multimedia presentations on viewers' information-processing activities measured by eye-tracking technology. Journal of Science Education and Technology, pp. 276286.

Civelek, T., Ucar, E., Ustunel, H., \& Aydin, M. K. (2014, June 10). Effects of haptic augmented simulation on K-12 students achievement and their attitudes towards physics. EURASIA Journal of Mathematics, Science \& Thecnology Education, pp. 565-574. 
Dan, A., \& Reiner, M. (2017, November 05). Reduced mental load in learning a motor visual task with virtual 3D method. Journal of computer assisted learning, pp. 1-10.

Di Serio, Á., Ibáñez, M. B., \& Kloos. (2015). IMpact of an augmented reality system os studentes motivation for avisual art course. Computers and Education, pp. 585-596.

Dillenbourg, P. (1999). What do you mean by collaborative learning. inCollaborative Learnning: COgnitive and Computing Approaches, 1-19.

Drachsler, H., Hung, Y., Chen, C., \& Huang, S. (2017). Applyying augmented reality to enhance learning: A study of diferente tezching materials. Journal of COmputer Assisted Learning, pp. 252-266.

Dunleavy, M., C., D., \& Mitchell, R. (2009). Affordances and limitations of immersive participatory augmented reality aimulations for teaching and learning. Journal of Science Educaton and Technology, pp. 7-22.

Dünse, A., R. G., \& Billinghurst, M. (2008). Asurvey of evaluation techniques used in augmented reality studies. Human Interface Technol. Lab.

Echeverria, A., Amestica, M. G., F., N. M., Barrios, E., \& Leclerc, S. (2012). Exploring different technological plataforms for suppporting co-located collaborative games in the clasroom. Computer in Human Behavior, pp. 1170-1177.

Estapa, A., \& Nadolny, L. (2015, September 15). The effect of an augmented reaity enhanced mathematics lesson on students achievement and motivation. Journal of STEM Education: Innovations and Research, pp. 4048.

Felizardo, K. R., Nakagawa, E. Y., \& Ferraz, S. C. (2017). Revisão Sistemática da Literatura em Engenharia de Software: Teoria e Prática. Rio de Janeiro: Elsevier.

Furió, D., Juan, M., Seguí, I., \& Vivó, R. (2015, May 22). Mobile Learning vc tradirional clasroom lessons: A comparative study. Jurnal of Computer Assited Learning, pp. 189-201.

Gog, T. v., \& Jarodzka, H. (2013). Eye tracking as a tool to study and enhance cognitive and metacognitive processes in computer-based learning environments. International handbook of metacognition and learning Technologies, pp. 143-156.

Google. (2017, Jan 20). Project Tango. Retrieved from https://get.google.com/tango/

Hase, S., \& Kenyon, C. (2001, February 21). Trove Partners. Retrieved from From Andragogy to Heutagogy: http://ultibase.rmit.edu.au/Articles/dec00/hase2.htm

Hollender, N., Hofmann, C., Deneke, M., \& Schmitz, B. (2010, June 26). Integrating cognitive load theory and concepts of human-computer interaction. Computers in Human Behavior, pp. 1278-1288.

Huang, Y., Li, H., \& Fong, R. (2016). Using augmented reality in early art education: A case study in Hong Kong Kindergaten. Early Child Development and Care, pp. 879-894.

Ibáñez, M. B., Á., D. S., Villarán, D., \& C, D. K. (2014). Experimenting witch eletromagnetism using augmented reality: Impact on flow student experience and educational effectiveness. Computer \& Education, p. 71.

Kamarainen, A. M., Metcalf, S., Grotzer, T., Browne, A., Mazzuca, D., Tutwiler, M. S., \& Dede, C. (2013, February 18). ECOMOBILE: INtegrating augmented reality and probeware with environmental education field trips. computers@Educations, p. 68. 
Kerawalla, L., Luckin, R., Seljeflot, S., \& Woolard, A. (2006). Expçoring the potenial of ugmented reality for tezching primary school science. Virtual Reality, pp. 163-174.

Kim, J., Marotta, K., Leo, J., Agarwal, S., Li, S., \& Chau, D. H. (2019, June 12-15). Mixed Reality for Learning Programming. Interaction Design and Children, pp. 574-579.

Kitchenham, B. (2019, June 17). Guidelines for performing systematic literature reviewsin software engineering. Retrieved from Keele University and University of Durham, U.K., Tech. Rep.: https://www.elsevier.com/_data/promis_misc/525444systematicreviewsguide.pdf

Klatzky, R. L., Wu, B., Shelton, D., \& Stetten, G. (2008, January). Effectiveness of Augmented-Reality Visualization versus Cognitive Mediation for Learning Actions in near Space. ACM Transactions on Applied Perception, p. 23.

Korbach, A., Brunken, R., \& Park, B. (2017). Measurement of cognitive load in multimedia learning: A comparison of different objective measures. Instrucional Science, 515-536.

Korbach, R., Brunken, R., \& Park, B. (2018). Differentiating different types of cognitive load: A comparison of different measures. Educational Psychology Review, 503-529.

Kucera, E., Haffner, O., \& Leskovský, R. (2018, Jan-Fev 31-03). Interactive and Virtual / Mixed Reality Applications for Mechatronics Education Developed in Unity Engine. 29th International Conference 2018 Cybernetics \& Informatics (K\&I), pp. 1-5.

Laine, T. H., Nygren, E., Dirin, A., \& Suk. (2016). Science spots AR: A plataform for science games with augmented reality. Education Technology Research and Development, pp. 507-531.

Lee, E. A., \& Wong, K. W. (2014). Learning with desktop virtual reality: Low spatial ability learners are more positively affected. Computers \& Education, 48-58.

Lindgren, R., Tscholl, M., Wang, S., \& Johnson, E. (2016). Enhacing learning and engagement through embodied interaction within a mixed reality simulation. Computer \& Education, pp. 174-187.

Maas, M. J., \& Hughes, J. M. (2020, March 13). Virtual, augmented and mixed reality in K-12. Retrieved from Technology, Pedagogy and Education Association: https://doi.org/10.1080/1475939X.2020.1737210

Madhukar, P., McCulloch, M., Gorman, J. D., Nitika, P., Enanoria, W., Kennedy, G., . . Colford, J. J. (2004, February 17). Systematic reviews and meta-analyses: an illustrated, step-by-step guide. he National medical, pp. 86-95.

Mahadzir, N. N., \& Phung, L. F. (2013, Jan/Fev 13-17). The use of augmented reality pop-up to increase motivation in Ensglish language for national primary school. Journal of Research \& Method in Education, pp. 26-38.

Microsoft. (2019, Jun 17). Microsoft Hololens. Retrieved from Microsoft Hololens: https://www.microsoft.com/microdot-hololens/en-g

Miligram, P., \& Kishino, F. (1994, December 25). A taxonomy of mixed reality visual displays. IEICE Transactions on Infomration and Systems, pp. 1321-1329.

Park, B., Korbach, A., \& Brunken, R. (2015). Do learner characteristics moderate the seductive-details-effect? A cognitive-load study using eye-tracking. Educational Technology and Society, 24-36.

Pass, F., Ayres, P., \& Pachman, M. (2008). Assessment of cognitive load in multimedia learning: Theory, methods, and applications. REcent innovations in educational technology that facilitate student learning , 11-35. 
Petersen, K., Vakkalanka, S., \& Kuzniarz, L. (2015). Guidelines for conducting systematic mapping studies in software engineering: An update. Information and Software Technology , pp. 1-18.

Piumsomboon, T., Day, A., Ens, B., Lee, Y., Lee, G., \& Billinghurst, M. (2017, November 27-30). Exploring Enhancements for Remote Mixed Reality. SA'17 Symposium on Mobile Graphics \& Interactive Applications, pp. 1-5.

Ralph, R., Jacoby, D., Coady, Y., Balachandar, D., Burt, E., \& Hnguyen, N. (2017, August 21-23). Metrics for Evaluation of Educational Experiences: Will Virtual Reality Have Impact? IEEE Pacific Rim Conference on Communications, Computers and Signal Processing (PACRIM), pp. 1-6.

Speicher, M., Hal, B. D., \& Nebeling, M. (2019, May 4-9). What is Mixed Reality? Conference on Human Factors in Computing Systems Proceedings (CHI 2019), pp. 1-15.

Sweller, J., Ayres, P., \& Kalyuga, S. (2011). Cognitive load theory. New York: Springer.

Sweller, J., Merriënboer, V., \& Paas, F. (2019). Cognitive architeutre and instrucional design: 20 years later. Educational Psychology Review, 1-32.

Tori, R., \& Hounsell, M. d. (2018). Introdução a Realidade Virtual e Aumentada. Porto Alegre: SBC.

Underwood, G., Jebbet, L., \& Roberts, K. (2004). Inspecting pictures for information to verify a sentence: Eye movements in general encoding and in focused search. . The Quartely JOurnal of Experimetnal Psychology Human Experimental Psychology, pp. 165-182.

Vasilevski, N., \& Birt, J. (2020, January 16). Analysing construction student experiences of mobile mixed reality enhanced learning in virtual and augmented reality environments. Research in Learning Technology, p. vol. 28.

Vygostky, L. (1987). Zone of proximal development. Mind in society: The develop,ent of higher psychological process.

Wazlawick, R. S. (2014). Metodologia de Pesquisa para Ciência da Computação. Rio de Janeiro: Elsevier.

Yannier, N., Hudson, S. E., \& Koedinger, K. R. (2020, February 03). Active Learning is About More Than Hands-On: A Mixed-Reality Al System to Support STEM Education. International Journal of Artificial Intelligence in Education, pp. 74-96.

Yoon, S. A., Elinich, K., W. J., Steinmeier, C., \& Tucker, S. (2012). Using augmented reality and Knowledge-building scaffods to improve learning in a science museum. International Journal of Computer-SUpported Collaborative Learning, pp. 156-168.

Zou, L., Tai, I., Covaci, A., Ibarrola, E., Ghine, G., \& Muntean, G. (2017, June 20-23). Canmultisensorial media improve learner experience. ACM Multimedia Systems Conf., Taipei, Taiwan, pp. 315-320.

Esta obra está bajo una Licencia Creative Commons Attribución-NoCommercial 4.0 International

(cc) BY-NC 